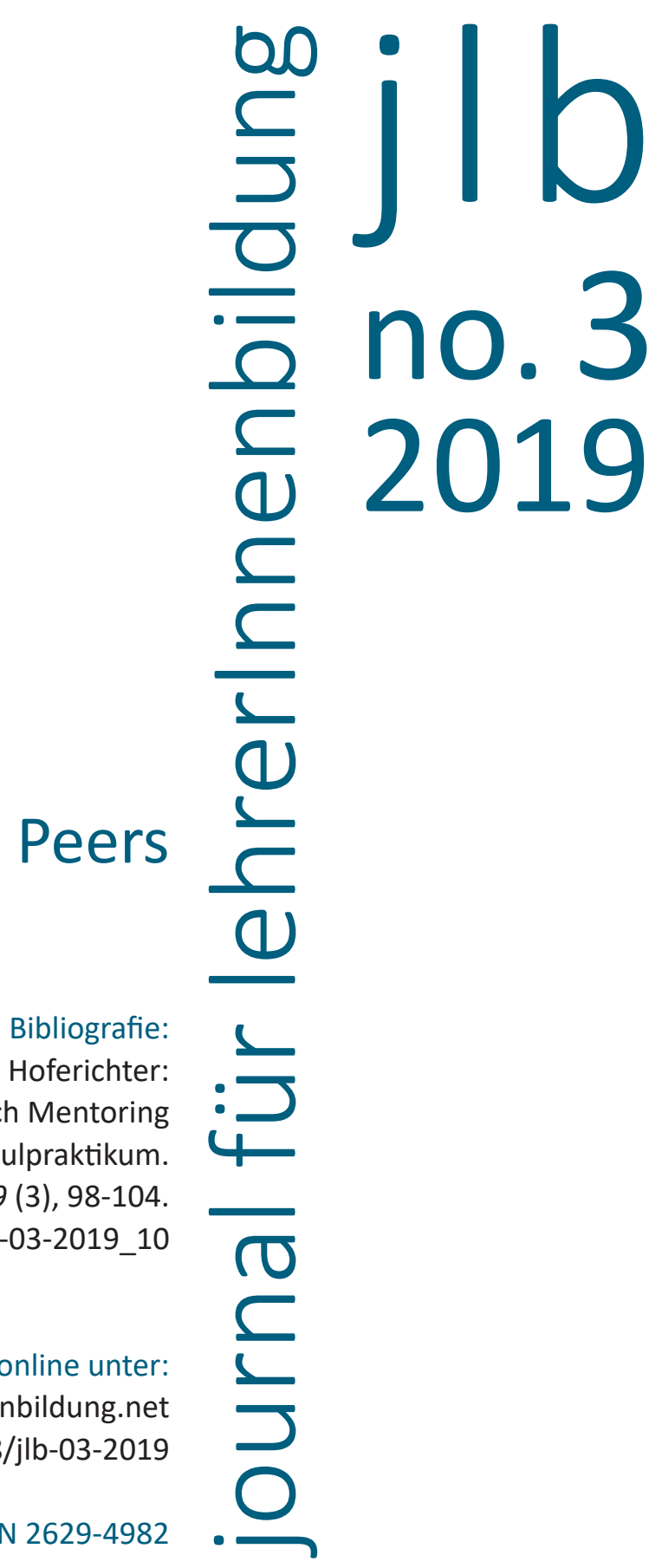


Frances Hoferichter

\section{Stressbewältigung durch Mentoring bei Lehramtsstudierenden im Schulpraktikum}




\section{Stress und Mentoring in der frühen Praxisphase}

Bereits während der Praxisphase fühlen sich Studierende hohen Belastungen durch die Arbeit an der Schule und ihr Studium ausgesetzt (Jantowski, Bartsch, Limmer \& Gumz, 2010). Befunde zeigen, dass sich Studierende wie auch Lehramtsanfänger*innen schlecht auf die Schulpraxis vorbereitet fühlen und in der Schule einen so genannten Praxisschock erleben (Süßlin, 2012).

Um der beschriebenen Belastung wie auch dem Praxisschock entgegenzuwirken, wurde an der Universität Greifswald eine reflexive Praxisphase inklusive Peer-Schul-Tandem-Mentoring eingeführt. Ziel dieser Praxisphase ist es, Studierende durch multiprofessionelle Begleitung auf die Berufspraxis vorzubereiten. Außerdem soll ein Peer-Schul- und Tandem-Mentoring die Studierenden dazu anregen, Strategien der Stressbewältigung wie positives Denken, aktive Stressbewältigung und soziale Unterstützung anzuwenden, um den hohen Belastungen durch das Praktikum und das Studium entgegenzuwirken. Die Peer-Mentor*innen sind Studierende, die das erste Schulpraktikum bereits absolviert haben. Tandempartner*innen sind Studierende, die zusammen mit einem anderen Studierenden das Schulpraktikum 1 an der Praxisschule absolvieren. Im Tandem-Mentoring sind beide Studierenden sowohl Mentee als auch Mentor*in und studieren nicht zwangsläufig dieselben Fächerkombinationen. Der/die Schul-Mentor*in ist ein*e Lehrer*in an der Praxisschule, der/die vor Ort als Koordinator*in, Ansprech- und Austauschpartner*in fungiert. Die Peer- und Schul-Mentor*innen wurden im Rahmen eines dreitägigen Mentoringworkshops zusammen ausgebildet. Im Workshop bearbeiteten die Teilnehmer*innen gemeinsam Themen wie Konfliktmanagement und -bewältigung, Teamarbeit und Kooperation, Mentoring- und Kommunikationsmodelle, Wahrnehmung, Reflexion sowie ein realistisches Rollenverständnis. Informationen zum ausführlichen Aufbau, den Inhalten sowie Materialien zum Mentoringworkshop sind derzeit in Arbeit.

\section{Unterstützt Mentoring die Stressreduktion?}

Bislang ist nicht untersucht, inwiefern ein multiprofessionelles Mentoring durch Peers, einen/einer Tandempartner*in und eine*n Lehrer*in 
an der Praxisschule die Strategien zur Stressbewältigung wie positives Denken, aktive Stressbewältigung und soziale Unterstützung von Studierenden in der ersten Praxisphase über den Verlauf des Praktikums aktiviert.

Aufbauend auf der Literatur (Raufelder \& Ittel, 2012) wird davon ausgegangen, dass Studierende, die von einem Mentoring durch einen Peer, Tandempartner*in und Lehrer*in begleitet werden, zunehmend Stressbewältigungsstrategien anwenden. Des Weiteren wird davon ausgegangen, dass insbesondere das Peer-to-Peer-Mentoring im Vergleich zum Schul-Mentoring, d. h. das Mentoring auf Augenhöhe durch einen Peer und eine*n Tandempartner*in alle drei Aspekte der Stressbewältigungsstrategien besonders stark aktiviert (Hoferichter \& Volkert, im Druck). In der vorliegenden Studie wird ein Modell getestet (s. Abb. 1), in dem sowohl der Zusammenhang des Peer-, Schul- und Tandem-Mentoring während des Schulpraktikums (1. MZP) mit den Komponenten der Stressbewältigung am Ende des Schulpraktikums (2. MZP) als auch dieser Zusammenhang unter Kontrolle der Stressbewältigung zum 1. MZP getestet wird.

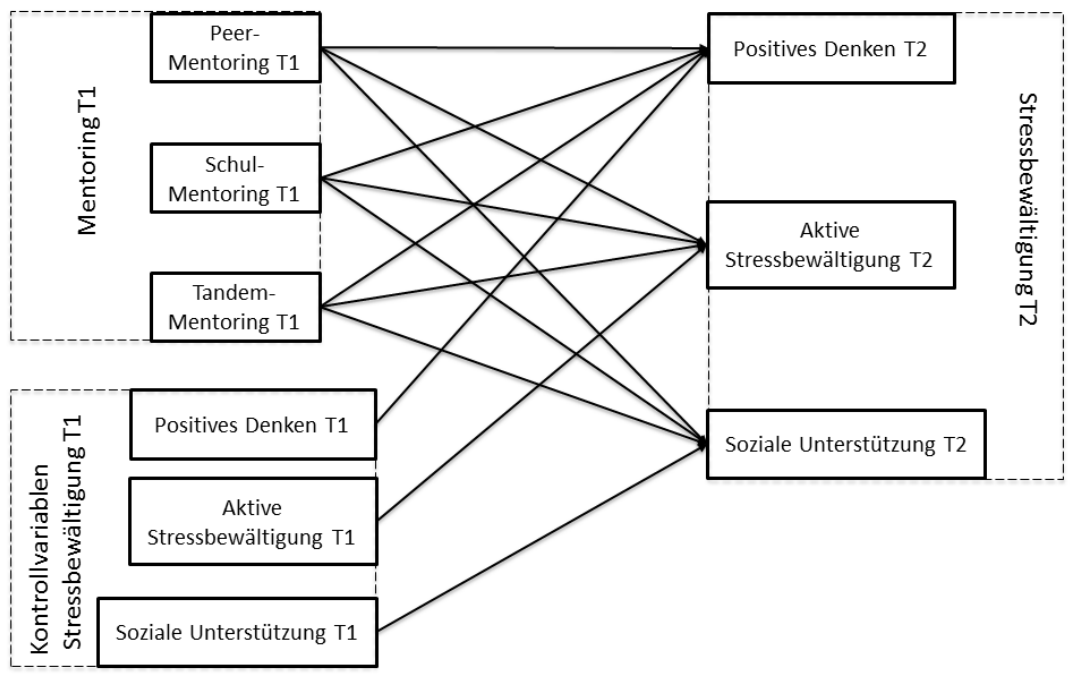

Abb. 1 Theoretisches Modell über die Beziehungen der Variablen Mentoring und Stressbewältigung 


\section{Methode}

\section{Teilnehmer*innen und Vorgehensweise}

Im Rahmen der "Qualitätsoffensive Lehrerbildung - LEHREN in M-V“ wurden im Jahr 2017/2018 insgesamt 26 Studierende (65 \% weiblich, MAlter $=24$, Altersspanne $=20-45$ Jahre, $S D=5,46)$ im Studiengang Lehramt an Gymnasien $(n=20)$ und Regionalen Schulen $(n=6)$ während und nach ihrem ersten Schulpraktikum mittels Fragebogen befragt.

\section{Messinstrumente}

Die Stressbewältigung (Satow, 2012) wurde anhand folgender Subskalen erhoben, mit jeweils vier Items auf einer 4-point Likert Skala von $1=$ trifft nicht $z u$ bis $4=$ trifft genau zu:

Positives Denken (MZP1 $\alpha=.70 ;$ MZP2 $\alpha=.60$ ), Aktive Stressbewältigung (MZP1 $\alpha=.76$; MZP2 $\alpha=.83$ ), Soziale Unterstützung (MZP1 $\alpha=.88$; MZP2 $\alpha=.84$ ). Das Mentoring (Eigenentwicklung) wurde anhand von folgenden Subskalen auf einer 4-stufigen Likert-Skala ( 1 = stimme nicht $z u$ bis $4=$ stimme voll $z u$ ) erhoben: Peer-Mentoring (sechs Items, $\alpha=.87$ ), Schul-Mentoring (sieben Items, $\alpha=.83$ ), Tandem-Mentoring (drei Items, $\alpha=.94$ ).

\section{Statistische Analysen}

Alle Analysen wurden mit dem Statistikprogramm Mplus, Version 8.1 durchgeführt. Zunächst wurde eine Pfadanalyse mit manifesten Variablen durchgeführt, in der die Beziehung der unabhängigen Variablen zum 1. MZP (Peer-Mentoring, Schul-Mentoring, Tandem-Mentoring) mit den abhängigen Variablen zum 2. MZP (positives Denken, aktive Stressbewältigung, soziale Unterstützung) getestet wurde. Anschließend wurden Kontrollvariablen (positives Denken, aktive Stressbewältigung, soziale Unterstützung zum 1. MZP) in das Modell aufgenommen.

\section{Ergebnisse und Diskussion}

Die Ergebnisse der Pfadanalyse zeigen (Tab. 1), dass Studierende, die im Sinne des Peer-to-Peer Ansatzes sowohl vom Peer-Mentoring als auch vom Tandem-Mentoring profitieren, eher Stressbewältigungsstrategi- 
en, wie z. B. positives Denken oder aktive Stressbewältigung anwenden. Profitieren Studierende von einem/einer Peer-Mentor*in, wenden sie eher eine aktive Stressbewältigung an. Dies gilt auch für das TandemMentoring - Studierende, die von einem Tandempartner profitieren, wenden eher aktive Stressbewältigung an wie auch positives Denken. Somit werden die Zusammenhänge zwischen Mentoring und Stressbewältigung besonders im Peer- und Tandem-Mentoring deutlich, was auf die emotionale Nähe und Statusähnlichkeit der Akteur*innen zurückzuführen ist (Hoferichter \& Volkert, im Druck). Tatsächlich beschreibt das similarity-attraction paradigm (Byrne, 1971), dass Individuen, die sich ähnlich sind, auch ähnliche Identitätsaspekte teilen, sich als Mitglieder einer "in-group" verstehen und sich gegenseitig bezüglich ihrer Interessen und Entwicklung beeinflussen bzw. voneinander lernen (Korte, 2007). Somit kann im Abgleich mit der Literatur und vor dem Hintergrund der vorliegenden Studie, der Peer-to-Peer Ansatz im Mentoring als Instrument gesehen werden, der Mentees nachhaltig in ihrer persönlichen und professionellen Entwicklung prägt.

Entgegen dieser Annahme berichten Studierende, die vom SchulMentoring profitieren, eher geringe Stressbewältigungsstrategien, d. h. wenig positives Denken, aktive Stressbewältigung und soziale Unterstützung, anzuwenden. Diese Ergebnisse scheinen zunächst überraschend, wenn man davon ausgeht, dass der/die Schul-Mentor*in den Studierenden beratend-begleitend zur Verfügung steht. Jedoch führt die vorliegende Mentee-Mentor*in-Beziehung dazu, dass Studierende weniger soziale Unterstützung erleben und darüber hinaus positives Denken und aktive Stressbewältigung anwenden. Dies mag daran liegen, dass der/die Schul-Mentor*in selbst wenig Stressbewältigungsstrategien anwendet und somit diese nicht an den Mentee weitergeben kann oder der/die Schul-Mentor*in sogar vom Schulalltag überfordert und resigniert ist (Leffers, 2003), was sich wiederum auf die Stressbewältigung der Mentees auswirkt. Immerhin leiden Lehrer*innen im Vergleich zu anderen Berufsgruppen in Deutschland am häufigsten unter Stress und Burnout (Scheuch, Haufe \& Seibt, 2015), was auch bedeutet, dass ihnen adäquate Stressbewältigungsstrategien im Repertoire fehlen. Um die Stressbewältigung von Studierenden im Schulpraktikum 1 mit Hilfe von Schul-Mentor*innen zu aktivieren, liegt es nahe, die Schul-Mentor*innen zunächst selbst und über einen längeren Zeitraum in der Anwendung von Bewältigungsstrategien zu schulen. 
Tab. 1 Ergebnisse der Pfadanalyse in Mplus, Modelle jeweils mit und ohne Kontrollvariablen

\begin{tabular}{|c|c|c|c|c|c|c|}
\hline \multirow[b]{3}{*}{ Prädiktoren } & \multicolumn{6}{|c|}{ Abhängige Variablen } \\
\hline & \multicolumn{2}{|c|}{$\begin{array}{c}\text { Positives Denken } \\
\text { T2 }\end{array}$} & \multicolumn{2}{|c|}{$\begin{array}{c}\text { Aktive Stress- } \\
\text { bewältigung T2 }\end{array}$} & \multicolumn{2}{|c|}{$\begin{array}{c}\text { Soziale } \\
\text { Unterstützung T2 }\end{array}$} \\
\hline & Est. (SE) & Est. (SE) & Est. (SE) & Est. (SE) & Est. (SE) & Est. (SE) \\
\hline $\begin{array}{l}\text { Peer-Mentoring } \\
\text { T1 }\end{array}$ & $\begin{array}{c}0,19 \\
(0,12)\end{array}$ & $\begin{array}{c}0,16 \\
(0,11)\end{array}$ & $\begin{array}{c}0,41 \\
(0,13)^{* *}\end{array}$ & $\begin{array}{c}0,23 \\
(0,14)\end{array}$ & $\begin{array}{l}-0,02 \\
(0,15)\end{array}$ & $\begin{array}{c}0,01 \\
(0,14)\end{array}$ \\
\hline $\begin{array}{l}\text { Schul-Mentoring } \\
\text { T1 }\end{array}$ & $\begin{array}{c}-0,51 \\
(0,17)^{* *}\end{array}$ & $\begin{array}{c}-0,55 \\
(0,16)^{* *}\end{array}$ & $\begin{array}{c}-0,47 \\
(0,18)^{* *}\end{array}$ & $\begin{array}{l}-0,32 \\
(0,18)\end{array}$ & $\begin{array}{c}-0,49 \\
(0,19)^{* *}\end{array}$ & $\begin{array}{c}-0,45 \\
(0,21)^{*}\end{array}$ \\
\hline $\begin{array}{l}\text { Tandem-Mento- } \\
\text { ring T1 }\end{array}$ & $\begin{array}{c}0,49 \\
(0,21)^{*}\end{array}$ & $\begin{array}{c}0,54 \\
(0,19)^{*}\end{array}$ & $\begin{array}{c}0,40 \\
(0,19)^{*}\end{array}$ & $\begin{array}{c}0,37 \\
(0,20)\end{array}$ & $\begin{array}{c}0,34 \\
(0,06)\end{array}$ & $\begin{array}{c}0,31 \\
(0,23)\end{array}$ \\
\hline \multicolumn{7}{|l|}{ Kontrollvariablen } \\
\hline $\begin{array}{l}\text { Positives Denken } \\
\text { T1 }\end{array}$ & & $\begin{array}{c}0,24 \\
(0,11)^{*}\end{array}$ & & & & \\
\hline $\begin{array}{l}\text { Aktive Stress- } \\
\text { bewältigung T1 }\end{array}$ & & & & $\begin{array}{c}0,45 \\
(0,11)^{* * *}\end{array}$ & & \\
\hline $\begin{array}{l}\text { Soziale } \\
\text { Unterstützung } \mathrm{T} 1\end{array}$ & & & & & & $\begin{array}{c}0,28 \\
(0,19)\end{array}$ \\
\hline $\mathbf{R}^{2}$ & 0,30 & 0,37 & 0,37 & 0,39 & 0,18 & 0,23 \\
\hline
\end{tabular}

Note, Berichtet werden standardisierte Regressionskoeffizienten (Est.), Standardfehler (SE), Signifikanzniveau bei ${ }^{*} p<0,05,{ }^{* *} p<0,01,{ }^{* *} p<0,00, T 1=1$. Messzeitpunkt T2 = 2. Messzeitpunkt

Zusammenfassend lässt sich festhalten, dass insbesondere das Peer- und Tandem-Mentoring als Form des Peer-to-Peer Ansatzes im Mentoring für Studierende während des ersten Schulpraktikums die Stressbewältigung begünstigt. Somit kann das Peer-to-Peer-Mentoring Studierende während der Praxisphasen in ihrer Stressbewältigung nachhaltig unterstützen.

Die Studie wurde im Rahmen der "Qualitätsoffensive Lehrerbildung LEHREN in M-V" vom Bundesministerium für Bildung und Forschung gefördert. Ich danke Prof. Dr. Dr. Diana Raufelder und Ute Volkert für Ihre Unterstützung im Projekt. 


\section{Literatur}

Byrne, D. (1971). The attraction paradigm. New York, NY: Academic Press.

Hoferichter, F. \& Volkert, U. (im Druck). (Praxis-)Reflexion durch multiprofessionelles Mentoring von Lehramtsstudierenden in der frühen Praxisphase. Herausforderung Lehrer_innenbildung - Zeitschrift zur Konzeption, Gestaltung und Diskussion.

Jantowski, A., Bartsch, A.-M., Limmer, J. \& Gumz, E. (2010). Evaluationsbericht zum Praxissemester im Jenaer Modell des Lehramtsstudiengangs - Belastungsempfinden und Studienzufriedenheit im modularisierten Lehramtsstudiengang unter Praxisbedingungen - Jena 2010. Verfügbar unter http://www.Ibf.uni-jena. de/zldmedia/Downloads/Forschung/Belastungsstudie_Evaluationsbericht.pdf [07.02.2019].

Korte, R. (2007). A review of social identity theory with implications for training and development. Journal of European Industrial Training, 31 (3), 166-180. doi: 10.1108/03090590710739250

Leffers, J. (10.04.2003). Stress im Klassenzimmer, Jeder dritte Lehrer ist ausgebrannt. Spiegel Online. Verfügbar unter http://www.spiegel.de/lebenundlernen/schule/ stress-im-klassenzimmer-jeder-dritte-lehrer-ist-ausgebrannt-a-244095.html [06.02.2019].

Raufelder, D. \& Ittel, A. (2012). Mentoring in der Schule - ein Überblick. Theoretische und praktische Implikationen für Lehrer/-innen und Schüler/-innen im internationalen Vergleich. Diskurs Kindheits- und Jugendforschung, 7 (2), 147-160.

Satow, L. (2012). Stress- und Coping-Inventar (SCI): Test-und Skalendokumentation. Verfügbar unter http://www.drsatow.de [07.02.2019].

Scheuch, K., Haufe, E. \& Seibt, R. (2015). Lehrergesundheit. Deutsches Ärzteblatt International, 112 (20), 347-356. doi: 10.3238/arztebl.2015.0347

Süßlin, W. (2012). Lehre(R) in Zeiten der Bildungspanik. Eine Studie zum Prestige des Lehrerberufes und zur Situation an den Schulen in Deutschland. Düsseldorf: Vodafone Stiftung Deutschland. Verfügbar unter https://www.vodafone-stiftung.de/ uploads/tx_newsjson/allensbach_04_2012.pdf [07.02.2019].

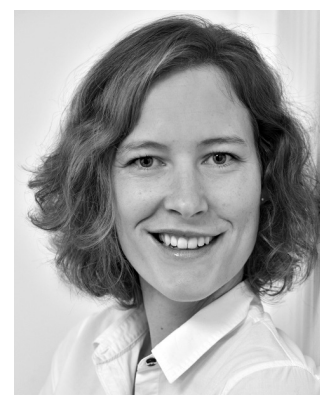

Frances Hoferichter, Dr., Vertr.-Prof. für Schulpädagogik an der Universität Greifswald. Arbeitsschwerpunkte: Mentoring, Stress, soziale Beziehungen

frances.hoferichter@uni-greifswald.de 